\title{
Padrão polínico utilizado por Tetragonisca angustula Latreille (Apidae: Meliponina)
}

Leila Nunes Morgado ${ }^{1,4}$, Rebeca Cássia Andrade ${ }^{1}$, Maria Cristina Affonso Lorenzon² e Vânia Gonçalves-Esteves ${ }^{3}$

Recebido em 14/02/2011. Aceito em 20/09/2011

\section{RESUMO}

(Padrão polínico utilizado por Tetragonisca angustula Latreille (Apidae: Meliponina)). O presente estudo objetivou quantificar e avaliar a carga polínica classificada por coloração encontrada nas corbículas de Tetragonisca angustula Latreille (Apidae: Meliponina). Os grãos de pólen encontrados nas corbículas de T. angustula no período estudado foram de 18 tipos polínicos pertencentes a 16 famílias vegetais. As cores predominantes indicaram uma diversidade de tipos polínicos. A coloração amarela obteve maior riqueza de espécies, sendo indicativo de preferência de cor pela T. angustula. A cor marrom foi a que apresentou a menor freqüência de grãos de pólen e a cor abóbora com a menor riqueza de tipos polínicos. As cores com tonalidades claras foram as mais representativas em número de grãos coletados. Os tipos polínicos mais freqüentes foram Meliaceae, Myrtaceae (Eucalyptus spp.), Piper mollicomum (Piperaceae), Schizolobium parahyba (Caesalpiniaceae) e Tibouchina granulosa (Melastomataceae).

Palavras-chave: abelha sem ferrão, coloração de pólen, interação planta-inseto, Palinologia

\begin{abstract}
(Pollen used by Tetragonisca angustula Latreille (Apidae: Meliponina)). The objective of this study was to quantify and evaluate the pollen load, which was classified by color, found on Tetragonisca angustula Latreille (Apidae: Meliponina). Eighteen pollen types, belonging to 16 plant families, were found on T. angustula. The majority of the pollen, from different species, was yellow, indicating that T. angustula prefers this color. This was followed by brown pollen and then pumpkin colored pollen. The pollen grains that were light colored were the most common. The more frequent pollen types were Meliaceae, Myrtaceae (Eucalyptus spp.), Piper mollicomum (Piperaceae), Schizolobium parahyba (Caesalpiniaceae) and Tibouchina granulosa (Melastomataceae).
\end{abstract}

Key words: Palynology, plant-insect interactions, pollen color, stingless bee

O grão de pólen é a principal fonte de nitrogênio para as abelhas e é coletado em quantidade nas fontes florais e estocado no alvéolo dentro de seus ninhos para a dieta da colônia. Diferentes métodos de investigação palinológica foram aplicados objetivando avaliar a fonte de recurso floral e sua utilização como alimento por esses himenópteros (Engel \& Dingemans-Bakels 1980, Biesmeijer \& Sommeijer 1992, Carvalho et al. 1999, Carvalho et al. 2001, Eltz et al. 2001, Leal et al. 2001; Barth 2004, Ramalho 2004, Alves et al. 2006, Gutiérrez \& García 2007; Barth et al. 2009).

De acordo com levantamento bibliográfico realizado são poucos os estudos que tratam da atratividade da coloração de grãos de pólen na coleta realizada pelas abelhas. Assim, objetivou-se quantificar e avaliar a carga polínica classificada por coloração encontrada nas corbículas de Tetragonisca angustula em bioma de Mata Atlântica.

\footnotetext{
${ }^{1}$ Universidade Federal de Lavras, Departamento de Entomologia, Lavras, MG, Brasil

${ }^{2}$ Universidade Federal Rural do Rio de Janeiro, Instituto de Zootecnia, Rio de Janeiro, RJ, Brasil

${ }^{3}$ Universidade Federal do Rio de Janeiro, Museu Nacional, Laboratório de Palinologia, RJ, Brasil

${ }^{4}$ Autor para correspondência: lelamorgado@yahoo.com.br
} 
Cinco colônias de T. angustula foram selecionadas na Ilha Grande/RJ (latitude de $23^{\circ} 11^{\prime}$ Sul e longitude de $44^{\circ}$ 12' Oeste) nos meses de fevereiro, março e abril de 2007. Em cada colônia foram capturadas abelhas campeiras, sendo retirados e separados por cor os grãos de pólen, totalizando um esforço amostral de 150 amostras.

Os tipos polínicos foram agrupados em quatro classes de freqüência relativa: pólen dominante (mais de $45 \%$ ), pólen acessório (entre 15 a 44\%), pólen isolado importante (entre 3 a 14\%) e pólen isolado ocasional (menos de 3\%) (Louveaux et al. 1970). Para a análise estatística foi utilizado o programa BioDiversity Pro (Mcaleece et al. 1997). O índice de diversidade foi o de Shannon-Wiener (H') (Magurran 1988) e o de similaridade foi de Cluster (Krebs 1989). As análises apresentaram nível de confiança de $\mathrm{R}^{2}=0,997\left(\mathrm{y}=0,2 \mathrm{x}^{2}+5 \mathrm{x}+2,3\right)$.

Totalizaram-se 14.346 grãos de pólen, sendo 6.253 de cor creme, 5.369 de cor amarela, 2.199 de cor abóbora e 525 de cor marrom. Os tipos polínicos encontrados nas corbículas de T. angustula no período estudado foram de 18 tipos polínicos pertencentes a 16 famílias vegetais. A cor amarela apresentou o maior índice de diversidade com 0,85 e a maior riqueza de espécies botânicas com 11 tipos polínicos, três tipos de pólen acessórios (entre 18,81 a 20,95 $\%$ ), quatro de pólen isolado importante (entre 9,35 a 9,95 $\%)$ e quatro de pólen isolado ocasional (menos de 1,6\%). A cor creme apresentou maior número de grãos, mas obteve um baixo índice de diversidade $(0,324)$, comparado com a cor amarela. A coloração marrom foi a que apresentou a menor abundância de grãos de pólen e a cor abóbora a menor riqueza de tipos polínicos. As cores com tonalidades claras foram as mais representativas em número de grãos de pólen coletados. As amostras de coloração marrom, creme e abóbora apresentaram tipos polínicos dominantes como Cecropia hololeuca (Cecropiaceae) com 85,71\%, Meliaceae com $75,85 \%$ e Schizolobium parahyba (Caesalpiniaceae) com $71,85 \%$, respectivamente (Tab. 1). A maior similaridade de tipos polínicos foi registrada na cor abóbora com a cor creme $(21,912)$.

Tetragonisca angustula visitou várias fontes de pólen, sendo freqüentes as famílias Melastomataceae, Myrtaceae, Piperaceae, Caesalpiniaceae, Meliaceae, Cyperaceae e Cecropiaceae com percentual acima de $15 \%$, confirmando o seu comportamento generalista. Ocorrência de tipos polínicos relacionados às espécies vegetais como Anadenanthera collubrina (Vell.) Brenam (Mimosaceae), Cecropia hololeuca Miq (Cecropiaceae), Myrtaceae (Eucalyptus spp.), Passiflora jilekii Wawra (Passifloraceae), Piper mollicomum Kunth (Piperaceae), Schizolobium_parahyba Vell (Caesalpiniaceae), Tibouchina granulosa Cogn. (Melastomataceae) e Trema micrantha Blume (Ulmaceae), também foram citados por autores como fontes de pólen para essa abelha (Iwama \& Melhem 1979, Carvalho et al. 1999, Carvalho \& Marchini 1999, Lorenzon et al. 2006).

A cor do pólen pode ser um indicativo de preferência na coleta de T. angustula, observando-se um maior número de grãos de pólen de cor clara como creme $(\mathrm{n}=6253)$, amarelo $(n=5369)$ e abóbora $(n=2199)$, contribuindo nas identificações de alguns tipos polínicos em dominância. Lau \& Galloway (2004) também sugerem a influencia da coloração do pólen de Campanula americana L. (Campanulaceae) na visitação de seus polinizadores, principalmente pelas abelhas da família Halictidae.

Tabela 1. Abundância (Abd), freqüência (\%), riqueza e índice de diversidade de tipos polínicos por coloração do pólen.

\begin{tabular}{|c|c|c|c|c|c|c|c|c|}
\hline \multirow{2}{*}{ Tipos polínicos } & \multicolumn{2}{|c|}{ Amarelo } & \multicolumn{2}{|c|}{ Abóbora } & \multicolumn{2}{|c|}{ Creme } & \multicolumn{2}{|c|}{ Marrom } \\
\hline & Abd & $(\%)$ & Abd & $(\%)$ & Abd & $(\%)$ & Abd & $(\%)$ \\
\hline Apocynaceae & 16 & 0,30 & - & - & - & - & - & - \\
\hline Araceae & - & - & - & - & 5 & 0,08 & - & - \\
\hline Arecaceae & - & - & - & - & - & - & 1 & 0,19 \\
\hline Asteraceae & - & - & - & - & - & - & 11 & 2,10 \\
\hline Caesalpiniaceae (Schizolobium parahyba) & 520 & 9,69 & 1580 & 71,85 & 926 & 14,81 & - & - \\
\hline Cyperaceae (Cyperus) & - & - & 520 & 23,65 & - & - & - & - \\
\hline Malpighiaceae (Tetrapterys) & 6 & 0,11 & - & - & - & - & - & - \\
\hline Melastomataceae (Tibouchina granulosa) & 1125 & 20,95 & - & - & - & - & - & - \\
\hline Meliaceae & 534 & 9,95 & - & - & 4743 & 75,85 & - & - \\
\hline Meliaceae (Trichilia) & 531 & 9,89 & - & - & 49 & 0,78 & - & - \\
\hline Mimosaceae & - & - & - & - & - & - & 12 & 2,29 \\
\hline Mimosaceae (Anadenanthera collubrina) & 86 & 1,60 & - & - & - & - & - & - \\
\hline Myrtaceae (Eucalyptus) & 1038 & 19,33 & - & - & - & - & - & - \\
\hline Passifloraceae (Passiflora jilekii) & - & - & 50 & 2,27 & - & - & - & - \\
\hline Piperaceae (Piper mollicomum) & 1010 & 18,81 & - & - & - & - & - & - \\
\hline Poaceae & 1 & 0,02 & 49 & 2,23 & - & - & - & - \\
\hline Ulmaceae (Trema micrantha) & 502 & 9,35 & - & - & - & - & 51 & 9,71 \\
\hline Cecropiaceae (Cecropia hololeuca) & - & - & - & - & 530 & 8,48 & 450 & 85,71 \\
\hline Riqueza & \multicolumn{2}{|c|}{11} & \multicolumn{2}{|c|}{4} & \multicolumn{2}{|c|}{5} & \multicolumn{2}{|c|}{5} \\
\hline Total / Abundância & \multicolumn{2}{|c|}{5369} & \multicolumn{2}{|c|}{2199} & \multicolumn{2}{|c|}{6253} & \multicolumn{2}{|c|}{525} \\
\hline Diversidade (Shannon H’ Log Base 10) & \multicolumn{2}{|c|}{0,85} & \multicolumn{2}{|c|}{0,325} & \multicolumn{2}{|c|}{0,324} & \multicolumn{2}{|c|}{0,234} \\
\hline
\end{tabular}




\section{Agradecimentos}

À Fundação Carlos Chagas Filho de Amparo à Pesquisa do Estado do Rio de Janeiro (FAPERJ) pela concessão da bolsa (Proc. E-26/150.557/2006, mtr. 2006.0927.5) a LNM. Ao Conselho Nacional de Desenvolvimento Científico e Tecnológico - CNPq pela concessão de bolsa de produtividade científica a VGE.

\section{Referências bibliográficas}

Alves, R.M.O.; Carvalho, C.A.L. \& Souza, B.A. 2006. Espectro polínico de amostras de mel de Melipona mandacaia Smith, 1863 (Hymenoptera: Apidae). Acta Science Biology 28(1): 65-70.

Barth, O.M. 2004. Melissopalynology in Brazil: a review of pollen analysis of honeys, propolis and pollen loads of bees. Scientia Agricola 61: 342-350.

Barth, O.M.; Munhoz, M.C. \& Luz, C.F.P. 2009.Botanical origin of Apis pollen loads using color, weight and pollen morphology data. Acta Alimentaria 38(1): 133-139.

Biesmeijer, K. \& Sommeijer, M.J. 1992. How to interpret pollen diets in bees? Proceedings of the Section Experimental and Applied Entomology The Netherlands Entomological Society 3: 210-215.

Carvalho, C.A.L. \& Marchini, L.C. 1999. Tipos polínicos coletados por Nannotrigona testaceicornis e Tetragonisca angustula (Hymenoptera, Apidae, Meliponinae). Scientia Agricola 56: 717-722.

Carvalho, C.A.L.; Moreti, A.C. de C.C.; Marchini, L.C.; Alves, R. M. \& Oliveira, P.C.F. 2001. Pollen spectrum of honey of "Uruçu" bee (Melipona scutellaris Latreille, 1811). Revista Brasileira de Biologia 61(1): 63-67.

Carvalho, C.A.L.; Marchini, L.C. \& Ros, P.B. 1999. Fontes de pólen utilizadas por Apis mellifera L. e algumas espécies de Trigonini (Apidae) em Piracicaba (SP). Bragantia 58(1): 49-56.
Eltz, T.; Brühl, C.A.; Kaars, S.V.D. \& Linsenmair, K.E. 2001. Assessing stingless bee pollen diet by analysis of garbage pellets: a new method. Apidologie 32: 341-353.

Engel, M.S. \& Dingemans-Bakels, F. 1980. Nectar and pollen resources for stingless bees (Meliponinae, Hymenoptera) in Surinam (South America). Apidologie 11: 341-350.

Gutiérrez, B.P. \& García, D.L.Q. 2007. Estudio melisopalinológico de dos mieles de la porción Sur Del Valle de México. Polibotánica 23: $57-75$

Iwama, S. \& Melhem, T.S. 1979. The pollen spectrum of the honey of Tetragonisca angustula angustula Latreille (Apidae, Meliponinae). Apidologie 10(3): 275-295.

Krebs, C.J. 1989. Bray-Curtis Cluster Analysis. 1. Biodiversity Pro Versão 2. Disponível em http://biodiversity-pro.software.informer. com/ (Acesso em outubro de 2010).

Lau, J.A. \& Galloway, L.F. 2004. Effects of low-efficiency pollinators on plant fitness and floral trait evolution in Campanula americana (Campanulaceae). Oecologia 141(4): 577-583.

Leal, V. M.; Silva, M. H. \& Jesus, N. M. 2001. Aspecto físico-quimico do mel de abelhas comercializado no município de Salvador- Bahia. Revista Brasileira de Saúde e Produção Animal 1(1): 14-18.

Lorenzon, M.C.A.; Conde, M.M.S. \& Barbosa, C.G. 2006. Eusocial Apidae in Tropical Insular Region. Brazilian Archives of Biology and Technology 49(5): 733-738.

Louveaux. J., Maurizio, A. \& Vorwohl, G. 1970. Methods of Melissopalynology. Bee Word 59: 139-157.

Magurran, A. 1988. Ecological diversity and its measurement. New Jersey, Princeton University Press.

Mcaleece, N.; Lambshead, P.J.D.; Paterson, G.L.J. \& Gage, J.G. 1997. Biodiversity professional. Beta-Version. London, The Natural History Museum and the Scottish Association for Marine Sciences.

Ramalho, M. 2004. Stingless bees and mass flowering trees in the canopy of Atlantic Forest: a tight relationship. Acta Botanica Brasilica 18(1): 37-47. 